\title{
User Selection and Signaling Over Time-Varying MIMO Broadcast Channels
}

\author{
Soroush Akhlaghi*, Amir K. Khandani* ${ }^{*}$ Abolfazl. Falahati ${ }^{\dagger}$ \\ *Electrical and Computer Engineering Department \\ University of Waterloo, Waterloo, ON, Canada \\ Email:\{soroush,khandani\}@cst.uwaterloo.ca \\ ${ }^{\dagger}$ Department of Electrical Engineering \\ Iran University of Science \& Technology (IUST) \\ Email:afalahati@iust.ac.ir
}

\begin{abstract}
Communication over Time-Varying Multiple Input Multiple Output (MIMO) broadcast channels with Zero-Forcing Beamforming (ZFBF) is considered. Different kinds of user selection algorithms are studied and the impact of channel time variation in the presence of outdated Channel State Information (CSI) is evaluated both analytically, and experimentally through Monte-Carlo simulation. In each user selection algorithm, $M$ beamforming directions are selected, where $M$ indicates the number of transmit antennas. Each active beamforming direction utilizes fixed-rate Block Coded Modulation (BCM) scheme. It is assumed that the perfect CSI is known at the beginning of each block, while the channel varies over time. The effect of channel aging is modeled as an additive noise with time-varying power. We introduce a new method based on Hadamard transformation in order to equalize the noise power across the coding block. The performance of BCM scheme is studied using the Pairwise Error Probability, showing that the proposed method results in a significant improvement in the error performance with a negligible increase in the complexity.
\end{abstract}

\section{INTRODUCTION}

Recent information theoretic results [1]-[3] show that multiple transmit antennas at a Base Station (BS) can be used to simultaneously transmit data to several users by converting the channel into data pipes that overlap in both time and frequency. It has been shown that the sum-capacity of MIMO broadcast channels can be achieved by applying Dirty-Paper Coding (DPC) at the transmitter [1]-[4]. However, existence results do not easily translate to practical implementation.

Beamforming is a suboptimal method with low complexity that achieves a large fraction of DPC performance [1]. Finding the optimal beamforming weight vectors, however, is a difficult task. Experimental results show that $M$ beamforming directions are adequate to achieve a significant portion of this capacity [7]. In this case, the optimum user selection requires the BS to perform an exhaustive search over the subsets of beamforming directions with cardinality $M$ which is computationally infeasible, especially for a large networks [8].

References [9], [10] use a very simple precoding scheme of Zero-Forcing Beamforming (ZFBF), where the weight vectors are chosen to avoid interference among users. This

This work is financially supported by Communications and Information Technology Ontario (CITO), Nortel Networks, and Natural Sciences and Engineering Research Council of Canada (NSERC). method selects a group of semi-orthogonal users in a greedy fashion, and then the beamforming weights are computed by inverting the corresponding channel matrix. As a result, the downlink is decomposed into $M$ orthogonal sub-channels between the BS and the uncoordinated receivers. This method has two shortcomings: (i) It wastes the transmit power as the channel matrix of the selected users maybe far from orthogonal, and (ii) It suffers from channel aging, resulting in interference between users. The current article addresses the above shortcomings by: (i) Selecting an appropriate set of users that are nearly orthogonal, and (ii) Applying a Hadamard transformation to the coding block to reduce the effect of the channel aging. The use of the Hadamard transformation averages the impact of the channel aging over all symbols in a given block. Relying on pair-wise error probability, we show that the use of the Hadamard transformation is nearly optimal in reducing the effect of channel aging.

This article is organized as follows: Section II contains a short overview and some background information on MIMO broadcast channels and user selection algorithms. In Section III, we investigate the effect of the channel aging in MIMO broadcast channels. Section IV discusses BCM and its performance evaluation for a time-varying channel and in order to overcome the effect of channel time variation, the proposed precoding strategy is introduced, as well. Numerical results are provided in Section V. Finally, Section VI concludes the paper. Throughout the paper, vectors are boldfaced lowercase and matrices are boldfaced uppercase, respectively. $x^{(i)}$ is the $i$ 'th element of vector $\mathbf{x}$ and $[\mathbf{X}]_{i, j}$ is the $(i, j)$ 'th element of matrix $\mathbf{X}$.

\section{SyStem MODEL}

We consider a MIMO broadcast channel with $M$ transmit antennas at the $\mathrm{BS}$ and $K(K \geq M)$ users with channel matrices $\mathbf{G}_{i} \in \mathcal{C}^{M \times N_{i}}$ for $i=1, \ldots, K$. The number of available beamforming directions for the $i$ 'th user is $r_{i}=$ $\min \left\{M, N_{i}\right\}$, where $r_{i}$ is the rank of the channel matrix of this user. Block Coded Modulation (BCM) and ZFBF is employed at the BS. It is assumed that perfect CSI is available at the start of the coding block, while the CSI varies over time. In order to have the minimum transmit power while the received power is restricted to be constant, the 
beamforming directions are selected to maximize $\operatorname{det}\left[\mathbf{H H}^{*}\right]$, where the matrix $\mathbf{H}$ is composed of the CSI of selected beamforming directions. Since searching for the best $M$ beamforming directions is computationally infeasible, the BS selects $M$ beamforming directions (among the total $\sum_{i=1}^{K} r_{i}$ beamforming directions) at time instant zero, indexed by $\pi(j), j=1, \ldots, M$, in a greedy fashion such that the corresponding vectors are nearly orthogonal and have a large norm. First, the maximum beamforming gains and the corresponding directions among all users are determined. The best user in terms of the maximum beamforming gain is selected and its beamforming direction is set along this direction. The algorithm proceeds in a recursive manner for remaining $M-1$ beamforming directions in such a way that in each step, the search for the best direction is performed in the null space of the previously selected users. The detail of the proposed algorithm is given in the following steps:

1) define the set of all beamforming directions as $\boldsymbol{\Gamma}=$ $\left\{\mathbf{v}_{1,1}, \ldots, \mathbf{v}_{1, r_{1}}, \ldots, \mathbf{v}_{K, 1}, \ldots, \mathbf{v}_{K, r_{K}}\right\} \in \mathcal{C}^{M \times 1}$. The first subscript denotes the corresponding user and the second subscript signifies the associated beamforming direction of this user. In what follows, for ease of notation, two subscripts are merged into the one subscript.

2) Select the beamforming direction with the highest norm as the first direction:

$$
\pi(1)=\arg \max _{i}\left\|\mathbf{v}_{i}\right\|, \mathbf{v}_{i} \in \boldsymbol{\Gamma}
$$

3) Choose the $m$ 'th beamforming direction as follows:

$$
\pi(m)=\arg \max _{i} \operatorname{det}\left[\boldsymbol{\Phi}_{m-1, i} \boldsymbol{\Phi}_{m-1, i}^{*}\right]
$$

where $\boldsymbol{\Phi}_{m-1, i}=\left[\mathbf{v}_{\pi(1)}|\ldots| \mathbf{v}_{\pi(m-1)} \mid \mathbf{v}_{i}\right]$, and $\left\{\mathbf{v}_{\pi(k)}\right\}_{k=1}^{m-1}$ is the set of selected beamforming directions up to the $(m-1)$ 'th step.

4) Set $\mathbf{H}_{0}=\boldsymbol{\Phi}_{M}$.

The BS transmits $M$ simultaneous coded data streams (with equal rate) to the selected beamforming directions by using a precoding matrix which is the inverse of the corresponding channel matrix. The mathematical model of the system at the $t$ 'th channel use is given by (the $i$ 'th row in this matrix equation corresponds to the $i$ 'th beamforming direction),

$$
\mathbf{y}_{t}=\mathbf{H}_{t} \mathbf{W} \mathbf{x}_{t}+\mathbf{n}_{t}, \quad 1 \leq t \leq T
$$

where $\mathbf{H}_{t} \in \mathcal{C}^{M \times M}$ and $\mathbf{W} \in \mathcal{C}^{M \times M}$ denote the channel matrix from the BS to the selected users and the beamforming matrix, respectively, $\mathbf{x}_{t} \in \mathcal{C}^{M \times 1}$ contains the transmitted signals, $\mathbf{y}_{t} \in \mathcal{C}^{M \times 1}$ contains the received signals, $\mathbf{n}_{t} \in$ $\mathcal{C}^{M \times 1}$ is circularly symmetric complex Gaussian noise with a zero-mean and unit-variance, i.e., $\mathbf{n}_{t} \sim \mathcal{N}_{c}\left(\mathbf{0}, \mathbf{I}_{M}\right)$, where $\mathbf{I}_{M}$ denotes the $M$ dimensional identity matrix. We assume flat, time correlated Raleigh fading, where the Jakes model is being used for numerical simulations.

The precoding matrix for ZFBF is selected according to the initial CSI, i.e.,

$$
\mathbf{W}=\mathbf{H}_{\mathbf{0}}^{*}\left(\mathbf{H}_{0} \mathbf{H}_{0}^{*}\right)^{-1}
$$

where (.)* denotes the conjugate transpose, and $\mathbf{H}_{0}$ is the channel matrix at time instant zero. For rectangular matrices, we have $\mathbf{W}=\mathbf{H}_{0}^{-1}$. We assume that $E\left[\mathbf{x x}^{*}\right]=P \mathbf{I}_{M}$.

The matrix $\mathbf{H}_{0}$ corresponds the initial CSI of the selected users, and can be uniquely decomposed as,

$$
\mathbf{H}_{0}=\mathbf{\Lambda R Q}
$$

where $\mathbf{Q}$ defines an orthonormal basis composed of vectors $\left\{\frac{\mathbf{q}_{\pi(1)}}{\left\|\mathbf{q}_{\pi(1)}\right\|}, \frac{\mathbf{q}_{\pi(2)}}{\left\|\mathbf{q}_{\pi(2)}\right\|}, \ldots, \frac{\mathbf{q}_{\pi(M)}}{\left\|\mathbf{q}_{\pi(M)}\right\|}\right\}$, and $\mathbf{R}, \boldsymbol{\Lambda}$ are lower triangular and diagonal matrices, respectively [10]. Matrix $\mathbf{R}$ is composed of elements,

$$
\mathbf{R}=\left(\begin{array}{ccccc}
1 & 0 & 0 & \ldots & 0 \\
\epsilon_{2,1} & 1 & 0 & \ldots & 0 \\
\vdots & \vdots & \ddots & \ldots & 0 \\
\epsilon_{M, 1} & \epsilon_{M, 2} & \ldots & \epsilon_{M, M-1} & 1
\end{array}\right)
$$

where the off diagonal elements are equal to,

$$
\epsilon_{i, j}=\frac{\mathbf{v}_{\pi(i)}^{*} \mathbf{q}_{\pi(j)}}{\left\|\mathbf{q}_{\pi(j)}\right\|} \quad 1 \leq i \leq M, 1 \leq j \leq i-1,
$$

Matrix $\boldsymbol{\Lambda}$ is composed of elements,

$$
\boldsymbol{\Lambda}=\operatorname{diag}\left\{\left\|\mathbf{q}_{\pi(1)}\right\|,\left\|\mathbf{q}_{\pi(2)}\right\|, \ldots,\left\|\mathbf{q}_{\pi(M)}\right\|\right\},
$$

where $\mathbf{q}_{\pi(i)}$ denotes the image of $i$ 'th beamforming direction in the null space of the previous selected directions.

Using the above matrix decomposition, the ZFBF precoding matrix is equal to,

$$
\mathbf{W}=\mathbf{H}_{0}^{-1}=\mathbf{Q}^{*} \mathbf{R}^{-1} \mathbf{\Lambda}^{-1},
$$

and the received signal is,

$$
\mathbf{y}_{t}=\mathbf{H}_{t} \mathbf{W} \mathbf{x}_{t}+\mathbf{n}_{t}, \quad 1 \leq t \leq T
$$

\section{The EFFect of ChANNEl AgING}

We use the following expression as statistical model for the time variations of the channel:

$$
\mathbf{H}_{t}=\rho_{t} \mathbf{H}_{0}+\sqrt{1-\rho_{t}^{2}} \mathbf{E}_{t}, \quad 1 \leq t \leq T,
$$

where $\rho_{t}$ denotes the correlation coefficient between the time instants 0 and $t, \mathbf{E}_{t}$ is a circularly symmetric complex Gaussian matrix with i.i.d entries, i.e. $\left[\mathbf{E}_{t}\right]_{i, j} \sim \mathcal{N}_{c}\left(0, \frac{1}{M}\right)$. It is assumed that the matrices $\mathbf{E}_{t}, t=1, \ldots, T$, are uncorrelated (independent). The received signal is equal to,

$$
\mathbf{y}_{t}=\rho_{t} \mathbf{x}_{t}+\sqrt{1-\rho_{t}^{2}} \mathbf{E}_{t} \mathbf{H}_{0}^{-1} \mathbf{x}_{t}+\mathbf{n}_{t} .
$$

Assuming that the correlation coefficient $\rho_{t}$ is known at the receivers, it follows:

$$
\begin{aligned}
\tilde{\mathbf{y}}_{t} & =\mathbf{x}_{t}+\frac{\sqrt{1-\rho_{t}^{2}}}{\rho_{t}} \mathbf{E}_{t} \mathbf{H}_{0}^{-1} \mathbf{x}_{t}+\frac{1}{\rho_{t}} \mathbf{n}_{t} \\
& =\mathbf{x}_{t}+\mathbf{i}_{t}+\frac{1}{\rho_{t}} \mathbf{n}_{t} .
\end{aligned}
$$

Since each active user knows only the corresponding beamforming direction, the matrix $\mathbf{H}_{0}$ is unknown at the receivers. As a result, the channel tracking techniques [12] are not applicable here and the second term in (13) is treated as an additive interference term.

Since the transmitted vectors $\mathbf{x}_{t}$ are uncorrelated, the resulting interference noise is uncorrelated across time [11]. 


\section{ERror PERformance}

As discussed earlier, the effect of aging can be modeled as a time-varying channel in which each active user experiences an extra additive noise term with unknown and varying power at each time instant. The baseband model of this system over one coding block is given by,

$$
\mathbf{r}=\sqrt{P} \mathbf{s}+\mathbf{z}
$$

where $\mathbf{s}, \mathbf{r}$ and $\mathbf{z}$ are $T$ dimensional vectors represented the transmitted, the received and the noise, respectively, and $E\left[\mathbf{z z}^{*}\right]=\operatorname{diag}\left[\sigma_{1}^{2}, \sigma_{2}^{2}, \ldots, \sigma_{T}^{2}\right]$. Let $\mathbf{c}$ and $\hat{\mathbf{c}}$ denote two distinct codewords, mapped to the modulation vectors $\mathbf{s}_{C}$ and $\mathbf{s}_{\hat{c}}$, respectively. The Pair-wise Error Probability (PEP), $\operatorname{Pr}\left(\mathbf{s}_{c} \rightarrow \mathbf{s}_{\hat{c}} \mid \mathbf{s}_{c}\right)$, is the probability that the transmitted modulation vector $\mathbf{s}_{c}$ is received incorrectly as $\mathbf{s}_{\hat{c}}$. Assume that $\mathbf{c}$ and $\hat{\mathbf{c}}$ have a Hamming distance $d(\mathbf{c}, \hat{\mathbf{c}})=k$ and let $\dot{\mathbf{s}}=\left[s_{c}^{\left(i_{1}\right)}, s_{c}^{\left(i_{2}\right)}, \ldots, s_{c}^{\left(i_{k}\right)}\right]$ and $\ddot{\mathbf{s}}=\left[s_{\hat{c}}^{\left(i_{1}\right)}, s_{\hat{c}}^{\left(i_{2}\right)}, \ldots, s_{\hat{c}}^{\left(i_{k}\right)}\right]$ denote the symbols of corresponding block in which the two modulation vectors are different. The receiver performs maximum likelihood detection by assuming a Gaussian noise with a constant power throughout the block (minimum distance decoding). Hence, the PEP can be evaluated as [11]:

$$
\operatorname{Pr}\left(\mathbf{s}_{c} \rightarrow \mathbf{s}_{\hat{c}} \mid \mathbf{s}_{c}\right)=Q\left(\frac{\sqrt{P}\|\mathbf{d}\|^{\mathbf{2}}}{2 \sqrt{\mathbf{d}_{r}^{T} \mathbf{M}_{d} \mathbf{d}_{r}+\mathbf{d}_{i}^{T} \mathbf{M}_{d} \mathbf{d}_{i}}}\right),
$$

where $\mathbf{M}_{d}$ is the correlation matrix of vector $\mathbf{z}$ in the positions that the two modulation vectors are different, and $\mathbf{d}=\mathbf{d}_{r}+j \mathbf{d}_{i}=\dot{\mathbf{s}}-\ddot{\mathbf{s}}$ is the corresponding difference.

For BPSK and QPSK modulation, the squared Euclidean distance between a pair of coded modulation vectors is proportional to the product of the constellation minimum Euclidean distance $\left(\mathcal{X}_{\text {min }}^{2}\right)$ and the Hamming distance between the two codewords $(k)$, i.e., $\|\mathbf{d}\|^{2}=k \mathcal{X}_{\text {min }}^{2}$.

Consider two channels, one with constant noise power across time and correlation matrix $\sigma^{2} \mathbf{I}_{T}$, and the second with an increasing noise power across time with correlation matrix $\operatorname{diag}\left[\sigma_{1}^{2}, \sigma_{2}^{2}, \ldots, \sigma_{T}^{2}\right]$. Also, $\sigma^{2}=\frac{\sum_{i=1}^{T} \sigma_{i}^{2}}{T}$ which means that the average energy of noise is the same in both cases. The average PEP for two codewords with Hamming distance $d(\mathbf{c}, \hat{\mathbf{c}})=k$ in the first channel is independent from the positions in which the two codewords are different. This depends on the Hamming distance as follows:

$$
P_{E_{1}}=Q\left(\sqrt{\frac{k \mathcal{X}_{\min }^{2} P}{4 \sigma^{2}}}\right) .
$$

For the channel with varying noise power, the average PEP in the presence of random interleaving would be,

$$
P_{E_{2}}=\frac{1}{\left(\begin{array}{l}
T \\
k
\end{array}\right)} \sum_{m=1}^{\left(\begin{array}{l}
T \\
k
\end{array}\right)} Q\left(\sqrt{\frac{k \mathcal{X}_{m i n}^{2} P}{4 \mu_{m}^{2}}}\right)
$$

where, $\mu_{m}^{2}=\frac{\sum_{j=1}^{k} \sigma_{m_{j}}^{2}}{k}$ is the average of noise power in the positions in which the two codewords are different (these positions are indexing by $m_{j}$ for $j=1, \ldots, k$ ). Denoting

$$
\begin{aligned}
\overline{\mu^{2}}=\frac{1}{\left(\begin{array}{c}
T \\
k
\end{array}\right)} \sum_{m=1}^{\left(\begin{array}{l}
T \\
k
\end{array}\right)} \mu_{m}^{2}, \text { one can see } & \\
\overline{\mu^{2}} & =\frac{1}{\left(\begin{array}{l}
T \\
k
\end{array}\right)} \sum_{m=1}^{\left(\begin{array}{l}
T \\
k
\end{array}\right)} \mu_{m}^{2}=\sum_{l=1}^{T} \frac{\left(\begin{array}{l}
T-1 \\
k-1
\end{array}\right) \sigma_{l}^{2}}{\left(\begin{array}{l}
T \\
k
\end{array}\right) k} \\
& =\sum_{l=1}^{T} \frac{\sigma_{l}^{2}}{T}=\sigma^{2}
\end{aligned}
$$

where (18) is computed relying on the observation that each value of the noise variance participate in exact $\left(\begin{array}{l}T-1 \\ k-1\end{array}\right)$ equations. Using (17), (18) and after some manipulations, it follows [11]:

$$
\begin{aligned}
P_{E_{2}} & \geq Q\left(\sqrt{\frac{k \mathcal{X}_{\text {min }}^{2} P}{4 \overline{\mu^{2}}}}\right) \\
& =Q\left(\sqrt{\frac{k \mathcal{X}_{\text {min }}^{2} P}{4 \sigma^{2}}}\right)=P_{E_{1}} .
\end{aligned}
$$

Equation (20) implies that in the case of the same average noise power, the performance of coded modulation techniques over an AWGN channel with fixed noise power across time results in a better performance as compared to the channel with different noise power.

It can be shown that applying Hadamard transformation to the vector of transmitted signals results in two benefits: (i) All the transmitted symbols will experience the same noise power, which is equal to the average noise power of the original channel. (ii) Relying on the central limit theorem, the resulting interference plus noise terms become nearly Gaussian, and hence, the common minimum distance decoding can be used for maximum likelihood detection.

In summary, the Hadamard transformation converts the channel into a new channel for which the total noise power across time remains constant. The resulting noise is no longer independent. However, simulation results show that this correlation is negligible in most situations.

\section{Numerical Results}

The performance of three kind of user selection algorithms are examined. We consider a broadcast channel with $M=$ 4 transmit antennas at the $\mathrm{BS}$ and $K=32$ users, each equipped with two receive antennas. $M=4$ beamforming directions are served at a given time and the information bits of each active user are first encoded by a Turbo code with block length 2048 and rate $\frac{1}{2}$, and then mapped to the QPSK constellation points through an interleaver. The resulting stream after applying the Zero-Forcing precoding matrix is transmitted through the channel. Figs. 1 and 2 show the performance of three different cases of user selection algorithms as follows: (i) Each user selects one of its antenna that has the better SNR ( Antenna selection), and hence, these $K$ beamforming directions are considered as the input of user selection algorithm introduced in section II. (ii) Applying Singular Value Decomposition (SVD) to the channel matrices of all users, with the restriction of applying at most one coordinate to any user ( method A). (iii) Applying SVD to all channel matrices and selecting the coordinates without any 
restrictions (method B). Note that in the case that each user has one receive antenna, the three user selection methods are the same. The plot of BER versus SNR for beamforming method in which the best user is only served at a time is given for comparison (TDMA ${ }^{1}$ ). In the beamforming method, the user with highest $\operatorname{det}\left[\mathbf{G G}^{*}\right]$ is selected and two 256QAM are transmitted for this user (for fair comparison in terms of fixed throughput). Fig. 3 shows the Frame Error Rate (FER) versus SNR for three different values of normalized Doppler frequency. The Jakes fading model is being used to simulate the time correlation of the underlying fading process and $M=4$ users, each equipped with one receive antenna are served at a given time. Simulation results show that for the case of Quasi-Static fading channel, i.e. $f_{d} T=0$, employing Hadamard transformation, does not result in any improvement. However, as the normalized Doppler frequency increases, the proposed scheme results in a remarkable improvement in the performance.

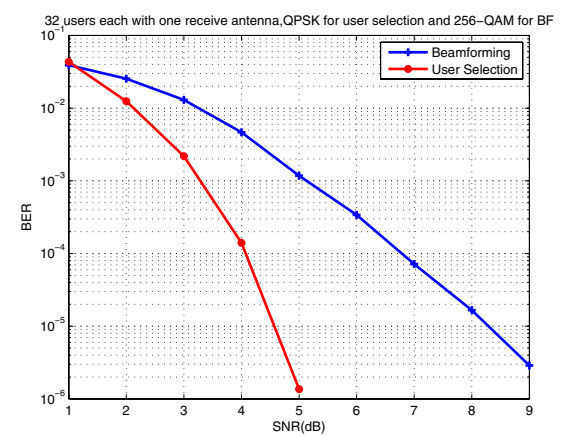

Fig. 1. The BER performance of user selection algorithm as compared to the conventional beamforming method for 32 users each with one receive antenna

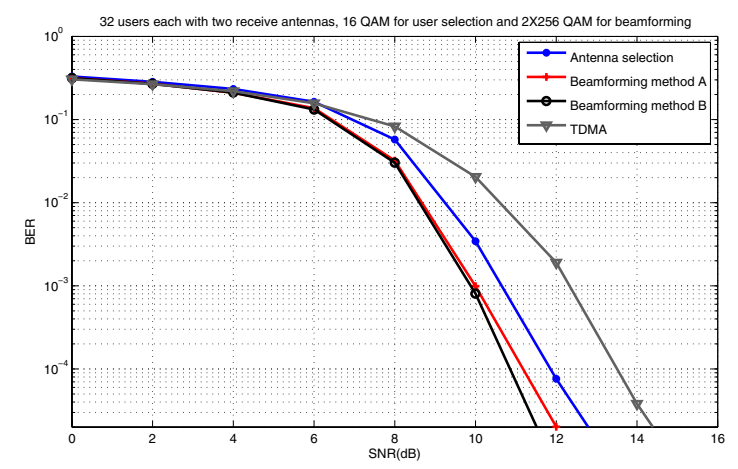

Fig. 2. The BER performance of different user selection algorithms for 32 users each with two receive antennas as compared to the conventional beamforming method

\section{CONCLUSIONS}

Three different types of user selection algorithms are examined and compared with conventional beamforming

\footnotetext{
${ }^{1}$ Time Division Multiple Access
}

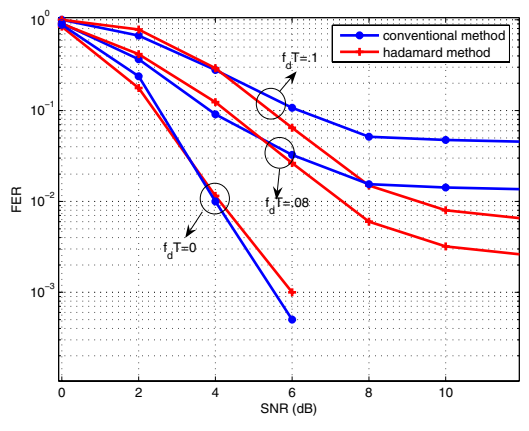

Fig. 3. The FER performance of proposed method as compared to conventional method

method. It is also shown that the impact of the CSI aging in MIMO broadcast systems with ZFBF at the transmitter can be modeled as a time varying channel in which the noise power varies across time. Then, the effect of coded modulation is studied for both the underlying channel and a channel with fixed noise power across time obtained by applying a Hadamard transformation. Analytical and simulation results show that the proposed algorithm results in a significant better performance as compared to the conventional method.

\section{REFERENCES}

[1] G. Caire and S. Shamai, "On the achievable throughput of a multiantenna Gaussian broadcast channel," IEEE Trans. Inform. Theory, vol. 49, pp. 1691-1706, July 2003.

[2] W. Yu and J. Cioffi, "Sum capacity of vector Gaussian broadcast channels," IEEE Trans. Inform. Theory, 2004.

[3] S. Vishwanath, N. Jindal, and A. Goldsmith, "Duality, achievable rates, and sum-rate capacity of Gaussian MIMO broadcast channels," IEEE Trans. Inform. Theory, vol. 49, pp. 2658-2668, Oct. 2003.

[4] P. Viswanath and D.N.C. Tse, "Sum capacity of the vector Gaussian broadcast channel and uplink-downlink duality," IEEE Trans. Inform. Theory, vol. 49, pp. 1912 - 1921, Aug. 2003.

[5] H. Hochwald and S. Vishwanath, "Space-time multiple access: Linear growth in the sum rate," Proceedings of 42 nd Allerton Conference, Allerton, IL, Oct 2002.

[6] P. Viswanath, D. Tse, and R. Laroia, "Opportunistic beamforming using dumb antennas," IEEE Trans. on Inform. theory, vol. 48, pp. 1277-1294, 2002.

[7] Mohammad Ali Maddah-Ali, Mehdi Ansari Sadrabadi, and Amir K. Khandani, "An effcient signaling scheme for mimo broadcast systems: Design and performance evaluation," IEEE Trans. on Info. Theory, Submitted for publication, 2005.

[8] S. Venkatesan and H. Huang, "System capacity evaluation of multiple antenna systems using beamforming and dirty paper coding," Bell Labs, unpublished.

[9] Q.H. Spencer, A.L. Swindlehurst, and M. Haardt, "Zero-forcing methods for downlink spatial multiplexing in multiuser MIMO channels,' IEEE Transactions on Acoustics, Speech, and Signal Processing, vol. 52, pp. 461-471, Feb. 2004.

[10] Taesang Yoo and Andrea Goldsmith, "On the optimality of multiantenna broadcast scheduling using zero-forcing beamforming," To appear: IEEE JSAC Special Issue on $4 G$ Wireless Systems.

[11] Soroush Akhlaghi, Amir. K. Khandani, and A. Falahati, "Reducing the effect of channel time variations in mimo broadcast systems," Technical Report is available at www.cst.uwaterloo.ca, 2006.

[12] j. R. Zeidler P. Yee S. Haykin, A. H. Sayed and P. C. Wei, "Adaptive tracking of linear time-varient systems by extended rls algorithms," IEEE Trans. Signal Processing, 1997. 